\section{Attention in infants as a function of magnitude of discrepancy and habituation rate ${ }^{1}$}

ROBERT B. MCCALL, Fels Research Institute, Yellow Springs, Ohio 45387, and WILLIAM H. MELSON, University of North Carolina, Chapel Hill, N.C. 27514

The distribution of attention to different magnitudes of discrepancy from a familiarized standard was studied in infant boys, $5 \frac{1}{2}$ months of age. Attention in terms of cardiac deceleration was found to vary as a function of magnitude of discrepancy, and the pattern was consistent with that predicted by the discrepancy hypothesis. Further, habituation to the repeatedly presented standard stimulus in terms of first fixation time (but not cardiac deceleration) predicted the extent of the infants' response to discrepancies as reflected in both of these response measures.

One methodological strategy in research on the distribution of attention in human infants has been to investigate the relative power of familiar and novel stimuli to recruit attention. Some authors have suggested that a simple dichotomy of novel and familiar stimuli may be an oversimplification, and that the magnitude of "novelty" (or the magnitude of "discrepancy" between the familiar standard and the new stimulus) is a controlling variable (Berlyne, 1960; Hunt, 1963). More specifically, the "discrepancy hypothesis" predicts attention to be an inverted-U function of discrepancy, and some statements of the theory propose that less attention (or even fear) may be elicited by extreme discrepanoies than by the familiar standard (e.g., Hunt, 1963).

There has been some empirical support for the proposition that the amount of attention will be a function of the magnitude of discrepancy (e.g., McCall \& Kagan, 1967, in press; Melson \& $\mathrm{McCall}^{2}$ ), but in each case the effect was limited either to one sex or to infants who displayed relatively rapid habituation of their attentional responses to the repeated presentation of the familiar standard. The fact that infants who demonstrate rapid habituation respond more to discrepancies suggests that rate of habituation may index the acquisition of some type of memory engram for the standard. Most of the

Fig. 1. The stimuli used in the study. From left to right and from top to bottom, the stimuli are referred to in the text as $A$, $B, C$, and D, respectively. demonstrations of such a relationship have measured habituation and the response to discrepancy in terms of the same behavioral index (e.g., visual fixation time). This fact makes these data prone to criticism that the relative response to discrepancy is determined by the amount of response given to the preceding standard and that rapidly habituating infants would have a lower value and thus be expected to give a higher relative response to the subsequent discrepancy.

The present study was designed to acquire more information on the tenability of the discrepancy hypothesis and to explore the prediction that rapid-habituating infants will respond to discrepancies with greater attention than will slow-habituating infants, and that this relationship is not an artifact of initial values.

\section{SUBJECTS}

Twenty-one male infants were recruited by calling mothers whose names were obtained from hospital records. The Ss averaged 146 days of age $(S D=7.85)$, and the mean education level of the parents (16 equals a college graduate) was 17.0
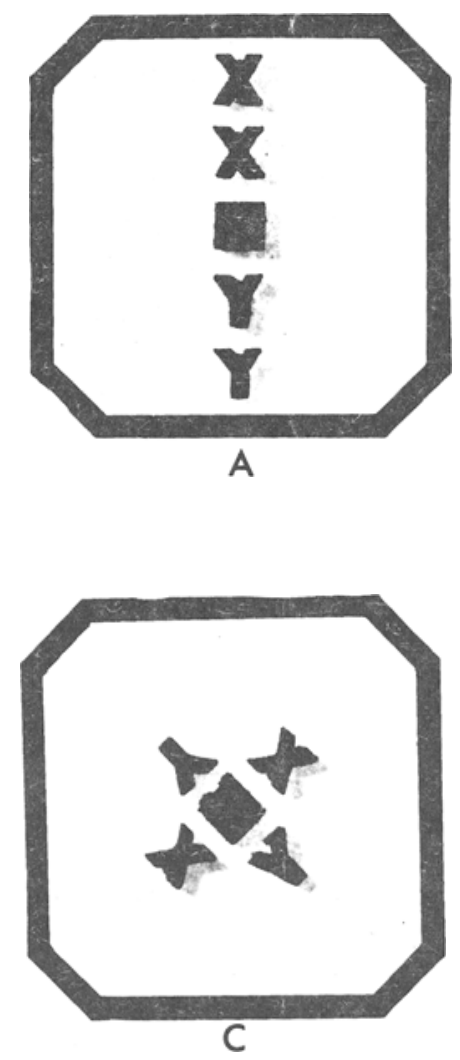

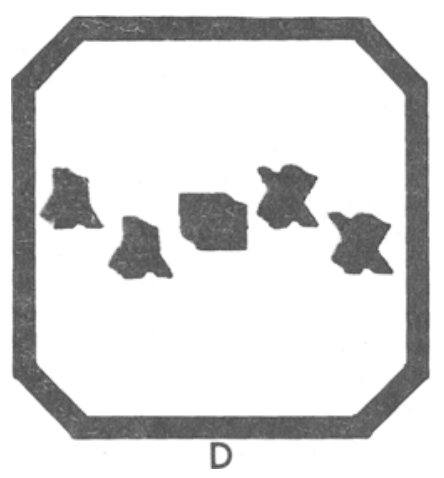

$(S D=1.78)$. All were normal Caucasian infants. The sample was restricted to boys because the girls in the community were being seen for a different observation. APPARATUS

The stimuli are illustrated in Fig. 1 and are identical to those used previously by McCall \& Kagan (1967). Stimulus A was the standard and was conceived to be a vertical linear form. The other stimuli varied from it, first in terms of verticality and then also in terms of linearity, so that an ordinal scale of discrepancy from $A$ was produced. McCall \& Kagan (1967) report that a sample of adults also perceived these stimuli on such a scale of discrepancy from A. The stimuli were $8 \times 8$ in. and were constructed out of Masonite painted white and trimmed in black. The three-dimensional Xs, Ys, and blocks were made of green Styrofoam.

The infant sat in a standard infant seat attached to a low table so that the eye-to-stimulus distance was approximately 30 in. The infant was surrounded on both sides and in front by a medium-gray three-sided enclosure, with each side-panel being $41 / 2 \times 7 \mathrm{ft}$, and the front panel, $5 \times 7 \mathrm{ft}$. The mother sat next to, but to the rear, of the infant. Ilumination was provided by a 150-W floodlight, located above and behind the infant, $4 \frac{1}{2} \mathrm{ft}$ from the stimulus. The front panel of the enclosure included a frame that could hold

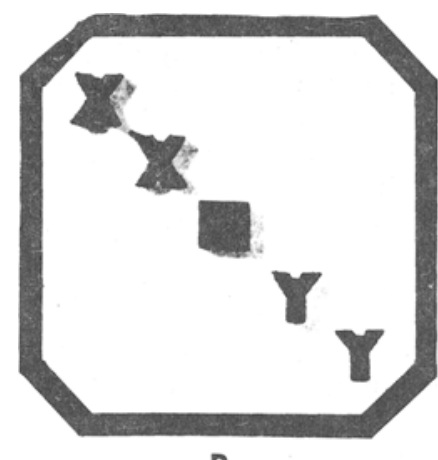

B 
the stimuli and that had two small windows located on either side through which the infant could be observed.

The dependent variable of first-fixation time was recorded by an $\mathbf{O}$ looking through the one-way window in the front panel of the apparatus. Heart rate was monitored with a set of Beckman bipotential electrodes and was recorded on a Beckman-Offner polygraph located in an adjoining sound-insulated room.

\section{PROCEDURE}

After the electrodes were placed on the baby (one over the left nipple, one below the right rib cage, and a ground near the navel) and he was positioned in the infant seat in front of the stimulus panel, the mother was asked to stand in front of him until the Es issued a "ready" signal (usually approximately $20 \mathrm{sec}$ ). Upon instruction, the mother sat in the chair provided and remained passive during stimulus presentations.

The stimuli were presented singly for $15 \mathrm{sec}$, with a 15 -sec interstimulus interval during which a blank white background was shown. The order of stimulus presentation was:

\section{S S S S S S S S D S S D S S D S}

in which $S$ was the standard, and D was a discrepancy. The 21 Ss were divided into three groups, the first $S$ to the first group, the second to the second, etc. Stimulus A (Fig. 1) was the standard stimulus under all conditions. The discrepant stimulus was Stimulus B (Group 1), C (Group 2), or D (Group 3), defining 1, 2, or 3 arbitrary units of discrepancy. The discrepant stimulus for any one $S$ was always the same stimulus $(B, C$, or D) on each presentation. Thus, each $S$ viewed only two different stimuli. Previous research (McCall \& Kagan, 1967) demonstrated that 4-month-old nonexperienced infants have no preferences among these four stimuli, and, thus, any differences in the attentional responses to $\mathrm{B}, \mathrm{C}$, and $\mathrm{D}$ were assumed to derive from the magnitude of discrepancy from $A$ that each represented.

The dependent variables of first-fixation time was defined to be the length of the first look (exceeding $1 / 2 \mathrm{sec}$ in duration) to a stimulus on any presentation. It was coded by an $\mathbf{O}$ who was unaware of the particular stimulus on any one trial. Previously estimated inter-O reliability was .93 (McCall \& Melson, in press). Cardiac deceleration was computed by comparing the mean of the three lowest adjacent beats during a fixation, with a baseline calculated from the rate during the $3 \mathrm{sec}$ prior to a fixation and the $10 \mathrm{sec}$ prior to stimulus onset. This procedure and its rationale are detailed elsewhere (McCall \& Kagan, 1967; McCall \& Melson, in press).
RESULTS

Magnitude of Discrepancy

Since the sample was small and measures of attention (particularly cardiac deceleration) were erratically distributed, nonparametric analyses were used throughout. By inspection, no major differences between the several levels of discrepancy were observed to occur across trials. The sum of the response to each of the three standard stimuli that preceded each of the three discrepancies was labeled $S$, and the sum of the response to the discrepant stimuli was called $D$. The major dependent variable was the simple difference (D-S), which reflected the response to discrepancy relative to the response to the preceding standard. Negative values of this difference indicated greater response to the standard than to the discrepancy, while positive values reflected greater response to the discrepancy than to the familiar standard. With respect to first fixation, a Kruskal-Wallis one-way analysis of variance on D-S for the three magnitudes of discrepancy was nonsignificant $(\mathrm{H}=1.63$, $\mathrm{df}=2 ; \mathrm{H}_{.05}=5.99$ ). Therefore, there was no evidence of a differential response in terms of first fixation to different magnitudes of discrepancy.

In contrast, a similar Kruskal-Wallis test on the cardiac measure (D-S) revealed a significant effect $(H=10.19, \quad d f=2$, $\mathrm{p}<.01$ ). These data are plotted in Fig. 2, in which the solid line represents the three points included in this analysis. The dashed line shows the relationship between these points and the response to the standard stimulus, which equals 0 because of the difference score (D-S) used as the dependent variable. Figure 2 tentatively suggests that this result conforms to the discrepancy hypothesis in that small

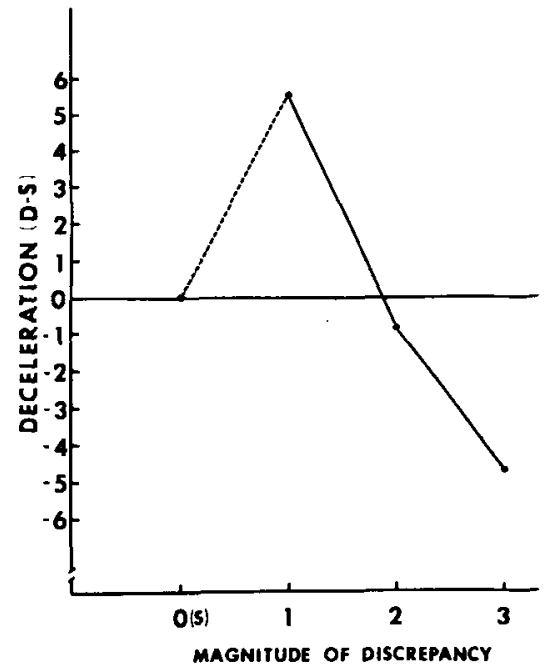

Fig. 2. The relative cardiac response to discrepancy as a function of the magnitude of discrepancy. discrepancies from the standard were responded to positively (more attention to the discrepancy than to the standard), but larger discrepancies were, in fact, responded to less than the standard was. In terms of individual Ss, all but one $S$ in the group experiencing one unit of discrepancy responded positively to the change, whereas all but one $S$ in the group receiving three units of discrepancy responded negatively to the discrepancy relative to the standard.

The Prediction of the Response to Discrepancy

An index of the rate of habituation over the first eight presentations of the standard was computed for both first fixation and deceleration by subtracting the response to the last two presentations of the standard from the first two presentations $\left(S_{1}+S_{2}-S_{7}-S_{8}\right)$. Positive values of this index indicated response habituation, whereas negative values reflected greater responses to later presentations of the standard than to early ones. In addition, the response-to-discrepancy measures (D-S) were adjusted for the fact that Ss received different discrepancy treatments by subtracting the mean of each treatment group from each of its scores. This yielded a residual score that was not influenced by group differences and that reflected the S's response to discrepancy. After these adjustments, rank-order correlations between the four measures revealed that habituation, in terms of first fixation, predicted the response to discrepancy both in terms of first fixation $(r=.75, p<.01)$ and cardiac deceleration $(\mathrm{r}=.52, \mathrm{p}<.05)$, whereas habituation in terms of the cardiac variable did not predict the response to discrepancy for either measure $(r=.08, .18)$.

\section{DISCUSSION}

These data indicate that, in short-term familiarization studies, the response to a novel or discrepant stimulus may be a function of the degree of discrepancy between that new stimulus and the familiarized standard. It can be seen from Fig. 2 that a simple dichotomy of familiar and novel stimuli would have yielded ambiguous results since the "novel stimulus" might have received an amount of attention that was greater than, equal to, or less than that given to the standard, depending upon the magnitude of discrepancy involved. In addition, although the study was not designed to test the full range of discrepancy including the standard, Fig. 1 does represent one of the first inclinations that the inverted- $U$ function predicted by the discrepancy hypothesis may have some validity in the context of infant attention (McCall, 1969). The fact that large discrepancies were attended to less than the familiar standard 
conforms to Hunt's (1963) summary of the discrepancy hypothesis and suggests that perhaps something like Sokolov's (1960) "defensive reaction" may function in the presence of such stimuli.

The finding that the rate of habituation predicts the response to discrepancy confirms previous observations (see McCall, 1969). The fact that the habituation of first fixation (but not deceleration) predicted both the fixation and the cardiac response to discrepancy seems to obviate the initial-value criticism since it would be difficult to explain the fixation-deceleration correlation in terms of initial values (especially since the deceleration-deceleration relationship was not significant).

Cardiac habituation did not predict cardiac response to discrepancy in this study, but such was the case when auditory stimuli were used (Melson \& McCall, $\left.1969^{2}\right)$. Perhaps the acquisition of a memory engram for a stimulus is more faithfully conveyed by the habituation of one response (i.e., first fixation) for visual stimuli and another response (i.e., cardiac deceleration) for auditory stimuli.

$$
\text { REFERENCES }
$$

BERLYNE, D. E. Conflict, anousal, and curiosity. New York: McGraw-Hill, 1960.

HUNT, J. McV. Motivation inherent in information processing and action. In $0 . J$.
Harvey (Ed.), Motivation and social interaction: Cognitive determinanta New York: Ronald Press, 1963. Pp. 35-94.

MOCALL, R. B. Maznitude of discrepancy and habituation rate as govemors of the attentional response of infants to new stimuli. Paper presented at SRCD, Santa Monica, 1969.

MOCALL, R. B., \& KAGAN, J. Stimulus-schems discrepancy and attention in the infant Journal of Experimental Child Psychology, 1967, 5, 381-390.

McCALL, R. B., \& KAGAN, J. Individual differences in the distribution of attention to stimulus discrepancy. Developmental Psychology, in press.

MOCALL, R. B., \& MELSON, W. Attention in infants as a function of complexity, contour, and area. Developmental Psychology, in press. SOKOLOV, E. N. Neuronal models and the orienting reflex. In M. A. B. Brazier (Ed.), The central nervous system and behavior. New York: Macy, 1960. Pp. 187-276.

\section{NOTES}

1. This research was supported by Grant HD-03110 to the University of North Carolina Child Development Research Institute, by USPHS Grants 05537 and 00222 to the Fels Research Institute, by Grants HD-03642 and HD-04160 to the first author, and by a grant from the Faculty Research Council at the University of North Carolina. The authors thank Lynne Christensen, Mary Chronister, Carl Corter, Christina Garratt, June Gregg, Jerome Kagan, Paul Obrist, Katherine Pryor, Anne Saravo, Pamela Srvoy, Roger Webb, and Miriam Young for their assistance and comments

2. Melson, W., \& McCall, R. B. The attentional responses of five-month infants to discrepant auditory stimuli. Unpublished manuscript, 1969.

\section{Correcting for compensation in studies of time estimation ${ }^{1}$}

\section{MYRON ARONS and HAR VEY LONDON, Brandeis University, Waltham, Mass. 02154}

When $E$ manipulates a variable in order to alter $S$ 's judgment of how much time has passed, $S$ may "compensate" for the effect of the variable upon him, i.e., he may revise his spontaneous judgment in order to accord with the reality demands of the situation, thus vitiating $E$ 's hypothesis. We propose a means of correcting for $S$ 's compensation and demonstrate its usefulness in an illustrative experiment on the effect of ego-involvement on time estimation.

We are sometimes interested in the extent to which a variable affects S's judgment of passing time. For instance, we might ask both bored Ss and interested Ss to estimate how long it took for a given length of time to pass. Given the evidence (Geiwitz, 1964; Loehlin, 1959; London \& Monello, in press), our expectation would be that the bored group's estimate will be longer.
We may, however, experience difficulty in confirming hypotheses about time estimation because of problems wholly extraneous to their validity. For example, Ss may "compensate" for the effect of the independent variable upon them. Suppose $S$ has been exposed to some set of stimulus conditions which, by hypothesis, have the effect of making time seem to pass slowly and is then given a questionnaire that asks him to estimate how much time passed. Even if E's hypothesis is correct, $\mathrm{S}$, given the possible reality demands of the situation, may go through something like the following thought process: "Well, it seemed like a long time passed-more than an hour, easily. However, I doubt very much that I've been here that long. It just doesn't seem possible that I've been here for over an hour. I guess I'll put down that $50 \mathrm{~min}$ passed." And thus, E's effort to alter S's time judgment is undercut.

This problem may be solved by straightforward questioning of $S$ about any compensation he may have done. We present below an illustrative study. in which the problem of compensation was anticipated and overcome.

\section{OVERVIEW}

Iverson \& Reuder (1956) have described a technique for manipulating ego-involvement. A number of studies (Meade, 1960, 1963; Rosenzweig \& Koht, 1933) have used this technique to show that time passes more quickly under conditions of increased ego-involvement.

Following these studies, Ss in our high ego-involved condition were led by instructions to believe that performance on a marble-sorting task would be indicative of creative potential, while $S s$ in the low condition were informed that participation in the task was merely for purposes of preexperimental testing of equipment. Time judgment was measured for each $S$ by the method of verbal estimation (MVE) (Bindra \& Waksberg, 1956) and corrected for compensation.

\section{SETTING AND EQUIPMENT}

The $S$ was required to sort marbles by color. A continuous flow of marbles was provided, with rate and order identical for all Ss. Several pseudo timing and recording devices were attached to the apparatus. $A$ wall mirror simulated a one-way obervation glass. Under the high ego-involved condition, this mirror was exposed to Ss; under the low ego-involved condition, it was covered. A tape recorder was concealed so that the revolution of the tape could provide no cues for time estimation.

\section{SUBJECTS}

Subjects were 30 paid female high-school students, aged 15 through 17 , recruited at their institutions. They were randomly assigned to the two experimental conditions, one-half to each.

\section{PROCEDURE}

The $E$ seated each $S$ and told her that she would perform a marble-sorting task and would receive a full explanation and instructions by tape recorder.

The $E$ then placed an opaque elbow-length glove over S's nonpreferred hand so that S would "not forget" to use her preferred hand. This ruse was devised to cover S's watch (all Ss in this experiment wore their watches on the nonpreferred hand) without arousing her suspicions about the purpose of the experiment.

As $E$ left the experimental room, he turned on the tape recorder. The recorder gave information in the following order: (a) the rationale of the experiment; (b) a review of instructions which were also on the wall to S's left; (c) a notice to await a starting buzzer before commencing the task; (d) a starting buzzer followed by $7.5 \mathrm{~min}$ $(450 \mathrm{sec})$ of recorded metronome beat, in 